\title{
SATS HVO Concept Validation Experiment
}

\author{
Maria Consiglio ${ }^{*}$, Daniel Williams ${ }^{\dagger}$, Jennifer Murdoch ${ }^{\ddagger}$, and Catherine Adams ${ }^{\S}$ \\ NASA Langley Research Center, Hampton, Virginia 23681
}

\begin{abstract}
A human-in-the-loop simulation experiment was conducted at the NASA Langley Research Center's (LaRC) Air Traffic Operations Lab (ATOL) in an effort to comprehensively validate tools and procedures intended to enable the Small Aircraft Transportation System, Higher Volume Operations (SATS HVO) concept of operations. The SATS HVO procedures were developed to increase the rate of operations at non-towered, non-radar airports in near all-weather conditions. A key element of the design is the establishment of a volume of airspace around designated airports where pilots accept responsibility for self-separation. Flights operating at these airports, are given approach sequencing information computed by a ground based automated system. The SATS HVO validation experiment was conducted in the ATOL during the spring of 2004 in order to determine if a pilot can safely and proficiently fly an airplane while performing SATS HVO procedures. Comparative measures of flight path error, perceived workload and situation awareness were obtained for two types of scenarios. Baseline scenarios were representative of today's system utilizing procedure separation, where air traffic control grants one approach or departure clearance at a time. SATS HVO scenarios represented approaches and departure procedures as described in the SATS HVO concept of operations. Results from the experiment indicate that low time pilots were able to fly SATS HVO procedures and maintain self-separation as safely and proficiently as flying today's procedures.
\end{abstract}

\section{Introduction}

$\mathrm{T}$ he SATS HVO concept of operations was developed to address the forecasted shortfall in capacity in the Air Traffic System. Its goal is to increase the utilization of non-towered, non-radar airports during periods of Instrument Meteorological Conditions (IMC) without a major negative impact to the Air Traffic Control (ATC) structure or workload. Fundamental principles of this concept such as the functional division of air traffic sequencing and separation tasks, a volume of controlled airspace around an airport, and ground based sequencing automation were part of a method of separation assurance described in [1]. The study included results of a batch simulation experiment of airport performance and provided a reference list of seminal works in the subject area. An evolved version of the concept was depicted in a later work [2] that added a modified notion of timed approaches to increase the number of concurrent Instrument Flight Rules (IFR) operations at similar airports. The SATS HVO Concept of Operations relies on a set of procedures, the establishment of a volume of airspace around designated airports where pilots accept responsibility for self-separation referred to as the Self Controlled Area (SCA) and a set of avionics that includes Automatic Dependent Surveillance-Broadcast (ADS-B) and air-ground datalink communications. A complete description of the procedures and required equipment can be found in [3, 4]. Flights operating in the SCA during IMC are given approach sequencing information computed by a ground based automated system referred as the Airport Management Module (AMM). Transition procedures between the SCA and controlled airspace can be found in [5].

The SATS HVO human in the loop validation experiment was conducted in the ATOL during the spring of 2004 to determine if a pilot can safely and proficiently fly an airplane while performing SATS HVO procedures. The

\footnotetext{
${ }^{*}$ Research Engineer, Crew Systems Branch, 20 West Taylor St., MS 152.

${ }^{\dagger}$ Research Engineer, Aviation Operations and Evaluation Branch, 20 West Taylor St., MS 152, AIAA Member.

${ }^{\ddagger}$ Research Psychologist, Aviation Operations and Evaluation Branch, MS 156A.

${ }^{\S}$ Research Psychologist, Crew Systems Branch, 20 West Taylor St., MS 152.
} 
experiment also sought to assess pilot workload and situation awareness using HVO procedures and tools. Comparative measures of flight path error, perceived workload and situation awareness were obtained for two types of scenarios. Baseline scenarios were representative of today's system utilizing procedural separation, where air traffic control (ATC) grants one approach or departure clearance at a time. SATS HVO scenarios represented approaches and departure procedures as described in the SATS HVO concept of operations [3]. Preliminary results from this experiment were published in [6]. The study also included a Monte Carlo analysis of throughput and approach delays that compared current day operations with SATS HVO. The same research objectives and experiment design were shared by a flight experiment conducted in the summer 2004 whose results can be found in [10]. The validation process included other studies that looked at different aspects of the concept. For example, in [7] the flight technical error metrics collected during simulation and flight experiments were compared in an effort to validate the simulation tools used in the ATOL. More recent pilot in the loop experiments investigated priority landings and visual flight rules (VFR) operations in the SCA as well as the use of some of the experimental avionics designed for the project $[8,9]$.

This paper provides a detailed description of the experiment design and results associated with subjective assessments of workload and situation awareness, procedure conformance and responses to usability questionnaires.

The organization of this paper is as follows: Section 1 provides a brief overview of the concept of operations. Section 2 introduces the experiment methodology, simulation platform, and the experiment design. Sections 3 and 4 contain the analyses of the experiment results and concluding remarks.

\section{Overview of the HVO Concept of Operations}

Key to the SATS HVO concept is the use of a newly defined area of flight operations called the SCA, established around SATS designated airports, (i.e., non-towered, non-radar equipped airports) during periods of IMC, similar to present day Class D Airspace that surrounds towered airports.

Within the SCA, pilots, using advanced airborne systems, would have the ability and responsibility to maintain separation between themselves and other similarly equipped airplanes. Aircraft operating in this airspace would need special avionics, e.g., automated dependent surveillance-broadcast (ADS-B), a two-way data link, and appropriate self-separation tools in order to participate. This concept would also require a new, ground-based automation system, the AMM, typically located at the airport that would provide appropriate sequencing information to the arriving aircraft. The AMM provides an arrival sequence and broadcasts the total number of arriving aircraft in the SCA. It does not, however, provide separation, altitude assignments, or sequence departures.

This proposed operational concept emphasizes the integration with the current and planned near-term National Airspace System by minimizing additional Air Traffic Control (ATC) workload. A simulation study conducted jointly by NASA LaRC and the Federal Aviation Administration (FAA) Technical Center focused on transition procedures between the SCA and ATC managed airspace. The study measured controller's workload and acceptability of SATS HVO operations [5]. Results from the experiment showed that no significant increase in controller's workload was observed during SATS HVO that resulted in a four-fold increase in operations.

The SATS HVO concept is a starting point or "template" for additional designs and analyses. No attempts have been made to optimize the size or shape of the proposed airspace. To date, the development focus has been on providing an operational concept that was safe, would enable more than one operation at a time, and would not require significant ground infrastructure costs or improvements. Global Positioning System (GPS) "T" instrument approach procedures were chosen as a basis for this concept, although other instrument approach procedures could be used as well.

Aircraft arriving into the SATS designated airport are managed by ATC according to an IFR flight plan to a transition fix above the SCA. The transition fix is also the Initial Approach Fix (IAF) on a GPS-T instrument approach procedure. Prior to reaching the transition fix, pilots must request landing sequence information from the AMM. The AMM message includes the SCA entry procedure (vertical or lateral), relative sequence information (follow <callsign>), and Missed Approach Holding Fix (MAHF) assignment. If the SCA is full, then the AMM sends a "stand by" message. An SCA configuration such as the one shown in Fig. 1 allows a maximum of four aircraft on approach ant any given time. The limit is based on the number of holding altitudes at the two MAHFs.

Pilots in the SCA initiate their approach once adequate spacing behind the lead aircraft has been met (determined through either a generic rule-based spacing procedure, i.e., safe for all combinations of aircraft performance, or by using an on-board self-spacing tool). For SATS HVO departures, pilots will file flight plans with a SATS HVO departure procedure to a Departure Fix (DF), obtain ATC clearance, and then use on-board information/tools to find a departure window, (e.g., safe separation from other approaching and departing traffic). 
Many of the features of the GPS-T based SATS HVO concept are depicted in Fig. 1. Two IAFs, Cathy and Annie, are shown that also serve as the MAHFs. There are also two DFs, Ginny and Ellen, located outside the SCA. SATS arrivals (Red and Blue aircraft) with alternating missed approaches, and departures (Green and Purple aircraft) are depicted in a "snapshot" in time:

- The blue aircraft is able to enter the SCA at the IAF approach altitude upon receipt of the AMM message: "IAF, lateral entry, follow none, missed approach Cathy". Lateral entries are permitted when no other aircraft are assigned to the same fix and follow none indicates there is no traffic to follow. Aircraft coordinates descent and release from ATC communication to initiate the approach. The missed approach is depicted as a blue dashed path.

- The red aircraft arrived by IFR clearance to the transition fix at $4000 \mathrm{ft}$ and received an AMM message that says: "IAF, vertical entry, follow blue aircraft (identified by registration number), missed approach to Annie". Vertical entries are required when there are other aircraft assigned to the fix or the approaching aircraft is too close for a lateral entry.

- The purple aircraft is departing the SCA via the DF ELLEN. The pilot contacted ATC to get a clearance and release time and initiated the departure when safe separation from the other aircraft in the SCA was determined.

- The green aircraft has already contacted ATC and gotten a clearance and release to depart

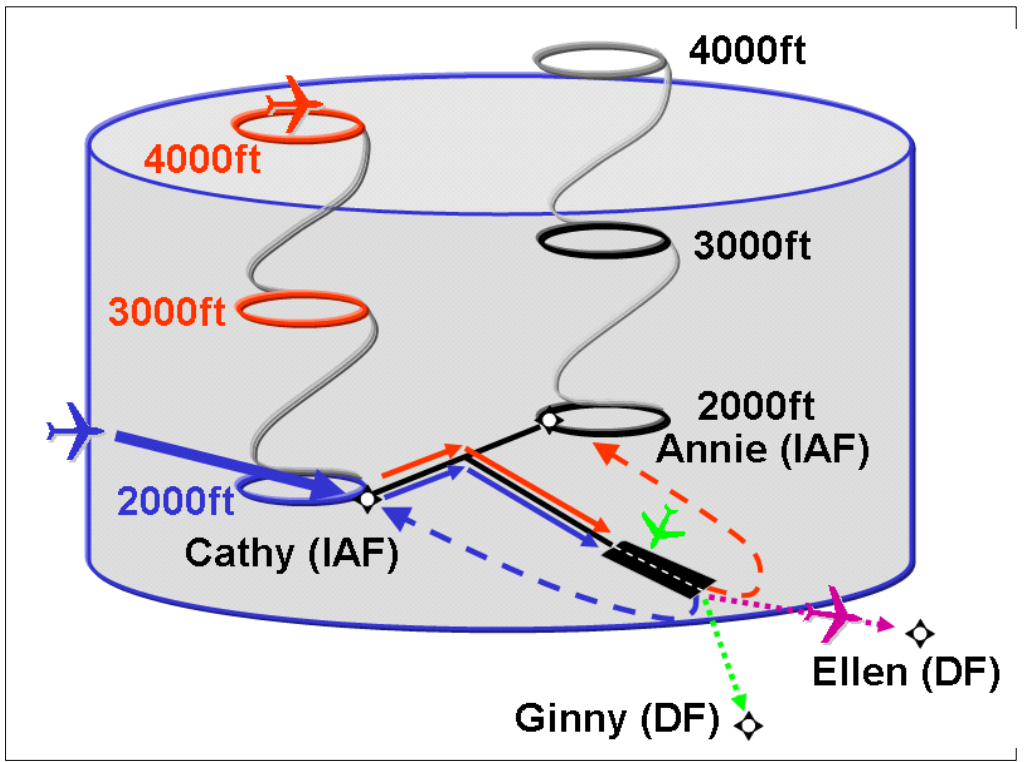

Figure 1. Schematic view of the SCA.

through DF GINNY. The aircraft is holding short and using on-board tools to find an open slot in the arrival stream to take the active runway and depart.

The piloted simulation experiment described in this paper was part of the validation and verification process of the SATS HVO concept that included multiple elements ranging from formal analysis of the procedures to a flight experiment, to full-system architecture prototype and public operational demonstration in June 2005.

\section{Validation Experiment Description}

\section{A. Objectives and Methodology}

In an effort to validate the concept from a pilot's workload perspective, this simulation experiment sought to answer the questions "Can pilots safely and proficiently fly an airplane while performing SATS HVO procedures?" and "Do pilots perceive that workload, while performing HVO procedures, is no greater than flying in today's system?". Following the simulation experiment, the same research objectives and design guidelines were shared by a flight experiment conducted during the summer of 2004. Results from that activity are reported in a separate document [10].

During the simulation experiment, evaluation pilots (EPs) were asked to manually fly a general aviation (GA) aircraft simulator during IMC while performing a series of approach and departure scenarios. Two types of scenarios were used:

1) Baseline scenarios were representative of current day IMC operations at non-radar equipped, non-towered airports (i.e., one operation at a time, managed by ATC). EPs were given departure and landing clearances as well as assigned altitudes and release instructions, and EPs were required to inform ATC of operation completion. 
Traditional flight instrumentation and an experimental multi-function display (MFD) were incorporated into the simulated cockpit. All scenarios were designed for Melfa airport in Accomack County (MFV), Virginia.

2) SATS HVO scenarios (Hereafter referred to as SATS scenarios) included only normal HVO operations and used the same starting location and traffic conditions as the Baseline scenarios. The same instrumentation and MFD were used during the SATS and Baseline scenarios.

The simulation experiment measured the flight technical error, perceived workload, and situation awareness of EPs throughout the operations associated with both the SATS and Baseline scenarios. Workload and situation awareness data were collected at the end of each scenario, and usability questionnaires were administered after the completion of all runs.

\section{B. Evaluation Pilots}

Evaluation Pilots (EPs) were randomly drawn from a pool of pilots that met a set of criteria. The EPs included fifteen instrument rated, male subjects, $50 \%$ with commercial certificates and $50 \%$ with private pilot certificates. All EPs were IFR rated and current. All EPs were high performance capable. One pilot had received their certified flight instructor rating. All EPs held less than 1000 hours total flight time. EPs experience included 10.9 hours using the GPS as a primary navigation instrument. All EPs had experience in simulators and 93\% had experience with a Horizontal Situation Indicator (HSI).

\section{Simulation Platform}

The simulation platform consisted of four pilot stations, a virtual traffic generator and an AMM with a plan view display. The pilot stations included a GA aircraft simulator, two flat panel displays for instrumentation, and one large plasma display for the out-thewindow view.

A yoke and trackball for flight control and instrument interaction and a set of head-phones/microphone allowed EPs to interact with other EPs and a simulated ATC station. All the pilot interface functionality designed for the SATS HVO scenarios was displayed in the experimental MFD shown in Figure 2. The MFD included a moving map with approach and path information, traffic and conflict detection and alerting symbology, procedure support

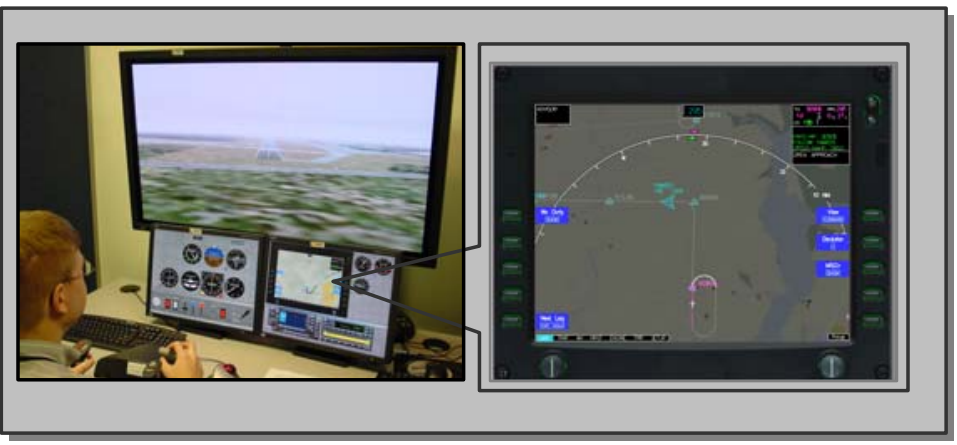

Figure 2. GA Pilot Station functionality and an AMM communications window used to request and receive sequencing information. A virtual traffic generator provided virtual traffic operating in the SCA environment responding to AMM sequencing by means of a pilot model that performed HVO flight procedures, responded to sequencing, and interacted with a simplified ATC model. The AMM provided sequencing information to all participating aircraft. A plan view display showed all the traffic in the SCA and vicinity (virtual traffic and pilot stations) and displayed the list of sequenced requesting aircraft. The ATC Model implemented required voice interactions for SCA transitions in SATS scenarios and approach and departure clearances in Baseline scenarios.

The operational environment included single pilot scenarios where a single EP interacted with virtual traffic and multiple pilot scenarios where four EPs participated in the same common scenario. Single pilot scenarios were more predictable while traffic conditions in multiple pilot scenarios had an added degree of variability and fidelity.

\section{Experimental Design}

As described above, two types of procedures were tested to compare the EPs' performance while completing both Baseline and SATS procedures. During SATS scenarios, EPs were required to follow SATS HVO approach and departure procedures. The pilot aircraft interface included the HVO experimental MFD with a moving map, approach and path information as well as AMM and procedure support information. During Baseline scenarios, EPs were required to follow today's approach and departure procedures, one operation at a time, in a simulated ATC managed airspace. The pilot interface included the experimental MFD with a moving map and approach and path information (AMM and procedure support information were not provided). Five different types of scenarios were designed for each procedure. They included departures, single and multiple aircraft approaches and missed 
approaches. There were 10 scenarios (i.e., five scenarios associated with Baseline procedures, and five scenarios associated with SATS HVO procedures).

Table 1: Experiment Design Matrix

\begin{tabular}{|c|c|c|c|}
\hline \multirow{4}{*}{} & \multicolumn{2}{|c|}{ PROCEDURE TYPE } \\
\cline { 2 - 4 } & Departure & $\mathrm{EP}_{1-15}$ & $\mathrm{EP}_{1-15}$ \\
\cline { 2 - 4 } $\begin{array}{c}\text { SCENARIO } \\
\text { TYPE }\end{array}$ & Approach Without Traffic & $\mathrm{EP}_{1-15}$ & $\mathrm{EP}_{1-15}$ \\
\cline { 2 - 4 } & Approach With Virtual Traffic & $\mathrm{EP}_{1-15}$ & $\mathrm{EP}_{1-15}$ \\
\cline { 2 - 4 } & $\begin{array}{c}\text { Approach to Missed } \\
\text { Approach with Virtual Traffic }\end{array}$ & $\mathrm{EP}_{1-15}$ & $\mathrm{EP}_{1-15}$ \\
\cline { 2 - 4 } & $\begin{array}{c}\text { Approach with Piloted Traffic } \\
\text { (Linked Simulation) }\end{array}$ & $\mathrm{EP}_{1-15}$ & $\mathrm{EP}_{1-15}$ \\
\hline
\end{tabular}

As shown in Table 1, the experiment design matrix used for data collection was a 2 (Procedure Type) x 5 (Scenario Type), within-subject design in which the same 15 participants (i.e., low time instrument rated pilots) were assigned to each experimental cell (i.e., test condition). The two independent variables used in the experiment design were procedure type and scenario type, and EPs 1 through 15 (EP 1-15) were asked to perform all 10 test conditions twice in partially counterbalanced order.

Dependent measures included EPs' flight technical errors, subjective workload levels, as assessed using the Modified Cooper Harper (MCH) Rating Scale [13], and situation awareness levels as assessed using the Situational Awareness Rating Technique (SART) [11].

\section{E. Experiment Scenarios}

Five different scenario types were designed that represented different approach and departure conditions. For each type two different procedures, Baseline and SATS were modeled.

- Departure with approaching traffic The pilot task was to receive an ATC clearance, taxi onto the active runway, takeoff, and depart the airfield. In Baseline scenarios, ATC waited to give departure clearance to the EP's aircraft until an approaching aircraft had landed and canceled its clearance. In HVO, the pilot was to selfdetermine when to depart by finding an opening in the approaching traffic flow. This was done by the pilot using a traffic display that showed aircraft on the approach and as long as the approaching aircraft was beyond the final approach fix (FAF), a departure was allowed.

- Approach without traffic (no holding required). The pilot task was to descend and fly the approach via ATC clearance during the Baseline scenarios. During the SATS scenarios, the pilot requested and received a "lateral entry, follow none" sequence from the AMM, received clearance to depart ATC space, and self-initiated the approach once in the SCA. Typically, the durations of the SATS scenarios were equivalent to those of the Baseline scenarios.

- Approach with virtual traffic (holding required). This scenario clearly differentiated Baseline from HVO. In Baseline, the pilot waited behind two other aircraft in holding until they had landed and ATC provided clearance to begin the approach (i.e., 30+ minutes in holding). In HVO, the pilot followed the AMM sequencing behind the two other aircraft, self-separated from the other aircraft in the SCA, and self-initiated the approach by following advisories provided by a self-spacing software tool. The SATS scenarios' durations were about half that of the Baseline scenarios.

- Approach to missed approach with virtual traffic (holding required). This scenario included having the pilot fly the missed approach. SATS scenarios required flying the missed approach while self-separating in the SCA. The SATS scenarios' durations were about two-thirds as long as the Baseline scenarios' durations.

- Multi-pilot linked simulation approaches (holding required). This scenario was flown with four EPs flying linked simulators to verify the HVO concept by introducing the variability of individually flown aircraft (instead of using one pilot in a scenario with precisely flown virtual traffic). The goal was to determine if pilots could conduct HVO while serving as traffic for one another. The time required to complete a multi-pilot HVO scenario was about one-quarter to one-third of the duration of a Baseline scenario. 


\section{Analysis of Results}

\section{A. Flight Technical Error (FTE)}

FTE, defined as the difference between the position estimated by the aircraft navigation system and the desired aircraft position, is a well-established measure of pilot performance that can reflect the effects of multiple factors such as increased workload or reduced situation awareness. In this study, airspeed, altitude and lateral path deviation were measured during the experiment to assess the FTE of EPs flying Baseline and SATS scenarios.

Airspeed, altitude and lateral path deviation were measured during the experiment to assess the FTE of pilots flying Baseline and SATS approach scenarios (departure scenarios were not assessed by FTE measures). Their task objective was to fly the scenarios while maintaining Instrument Rating Practical Test Standards (PTS) criteria

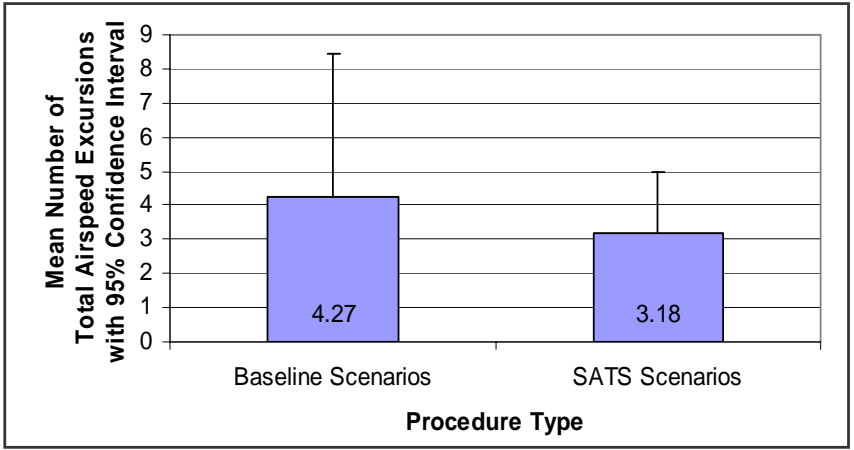

Figure 3. Mean Number of Airspeed Excursions [14]. Data were analyzed by way of repeated measures Analysis of Variance (ANOVA) tests, with the main effect of procedure type being of primary interest, set a priori at a 5-percent significance level $(\alpha=.05)$ [15].

For every flight path parameter, the results obtained supported the experiment hypothesis that EPs' flight performance during the SATS scenarios would be no worse than their flight performance during the Baseline scenarios. In some cases, results revealed that EPs' flight performance during the SATS scenarios was significantly better than their flight performance during the Baseline scenarios.

Airspeed: EPs were instructed to fly within the same airspeed envelope during the SATS and Baseline scenarios. The speed ranges for the initial, intermediate and final segments were 130 to 90 knots (kts), 110 to $90 \mathrm{kts}$ and 105 to $85 \mathrm{kts}$, respectively. As outlined below, three metrics were collected regarding airspeed.

Number of excursions outside the airspeed envelope: A statistically significant difference was found to exist between the total number of airspeed excursions associated with each procedure type as shown in Fig. 3. During the Baseline scenarios, 4.27 airspeed excursions

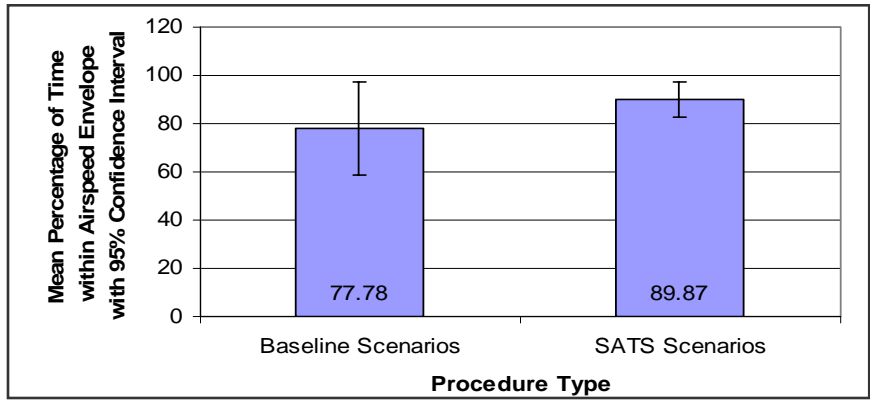

Figure 4. Percentage of time within airspeed envelope occurred [Mean $(M)=4.27$, Standard Deviation $(S D)=4.15$, Sample Size $(N)=60$ ]. During the SATS scenarios, 3.18 airspeed excursions occurred $(\mathrm{M}=3.18, \mathrm{SD}=1.81, \mathrm{~N}=60)$. This finding indicates that fewer airspeed excursions occurred when EPs performed the SATS than when they performed the Baseline scenarios.

Percentage of time within the airspeed envelope: As shown in Fig. 4, a statistically significant difference was found to exist between the percentages of time that EPs flew within the assigned airspeed envelope during each procedure type. During the Baseline scenarios, EPs flew within the defined airspeed envelope $77.78 \%$ of the time $(\mathrm{M}=$ 77.78, SD = 19.27, $\mathrm{N}=60$ ). During the SATS scenarios, EPs flew within the defined airspeed envelope $89.87 \%$ of the time ( $\mathrm{M}=$ 89.87, $\mathrm{SD}=7.43, \mathrm{~N}=60$ ). This finding indicates that EPs maintained airspeed within an assigned envelope more accurately when

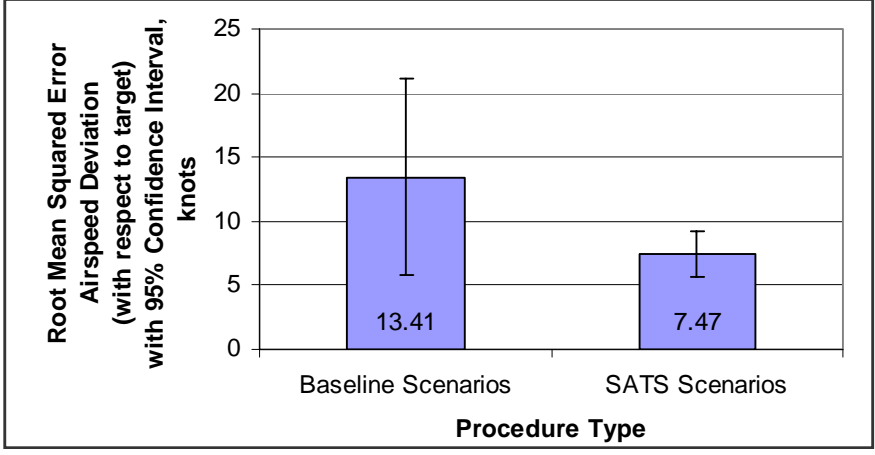

Figure 5. RMSE Airspeed with respect to target 
they performed the SATS scenarios than when they performed the Baseline scenarios.

RMSE with respect to airspeed target: A statistically significant difference was found to exist between the RMSE airspeed values associated with each procedure type as shown in Figure 7. A RMSE airspeed deviation of 13.41 kts occurred during the Baseline scenarios $(\mathrm{M}=13.41, \mathrm{SD}=7.65, \mathrm{~N}=60)$, and a RMSE airspeed deviation of 7.47 kts occurred during the SATS scenarios $(\mathrm{M}=7.47, \mathrm{SD}=1.79, \mathrm{~N}=60)$. This finding indicates that EPs maintained airspeed with respect to an assigned target value more accurately when they performed SATS scenarios than when they performed Baseline scenarios.

Altitude: EPs were instructed to fly within the same altitude envelope for both Baseline and SATS scenarios, +/$100 \mathrm{ft}$ of hold altitudes, $-100 \mathrm{ft}$ of "at or above" altitudes, and +100; $-0 \mathrm{ft}$ for minimum descent altitude (MDA) until missed approach point or visual transition to landing. This equates to: Initial Segment: Not below $1900 \mathrm{ft}$. Intermediate Segment: [Aircraft altitude at IF $+100 \mathrm{ft}, 1400 \mathrm{ft}$ ]. Final Segment: [Aircraft altitude at FAF + $100 \mathrm{ft}$, MDA]. A statistically significant difference was not found to exist between the number of excursions outside the altitude envelope or between the percentage of time within the altitude envelope that occurred during the SATS scenarios as compared with the Baseline scenarios.

Lateral path deviation: EPs were instructed to use target path as flight path for all segments using a target speed of $110 \mathrm{kts}$ for turns during both the Baseline and the SATS scenarios.

RMSE with respect to lateral path: A statistically significant difference was found to exist between the lateral path deviation RMSE values associated with each procedure type as shown in Fig. 6. A RMSE lateral path deviation of $0.12 \mathrm{~nm}$ occurred during the Baseline scenarios $(\mathrm{M}=$ $0.12, \mathrm{SD}=0.19, \mathrm{~N}=60$ ), and a RMSE lateral path deviation of $0.05 \mathrm{~nm}$ occurred during the SATS scenarios $(\mathrm{M}=0.05, \mathrm{SD}=0.03, \mathrm{~N}=60)$.

This finding indicates that EPs maintained lateral path deviation with respect to an assigned target value more accurately when they performed the SATS scenarios.

\section{B. Subjective Assessments of Workload}

EPs used the MCH Rating Scale to rate the level of workload that they experienced during each of the experiment's 10 test conditions.

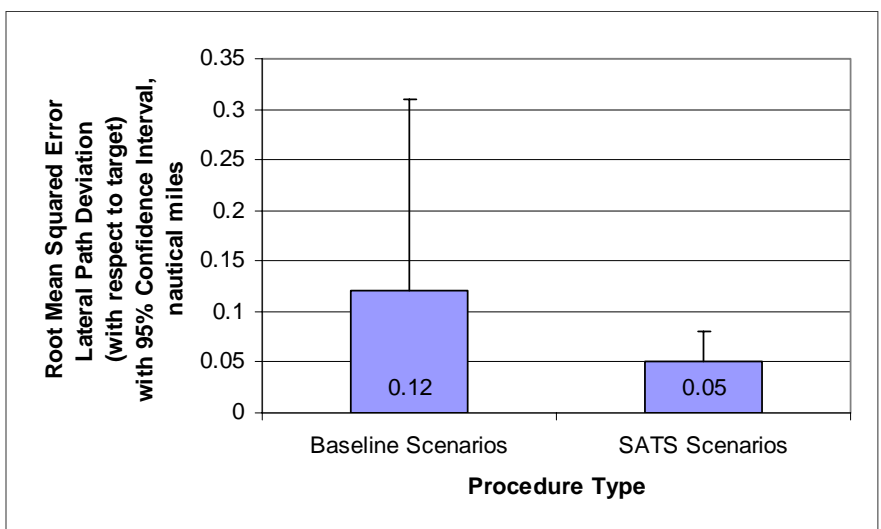

Figure 6. RMSE Lateral Path Deviation with respect to Target Path

Workload ratings could range on a scale from "1" (i.e., the instructed task was very easy/highly desirable; operator mental effort was minimal; and desired performance was easily attainable) to "10" (i.e., the instructed task was impossible; it could not be accomplished reliably). As reported below, nonparametric tests were employed as a conservative method for analyzing workload ratings associated with discrete rating scale items.

EPs reported experiencing a mean workload rating of 1.69 when performing the SATS procedures $(\mathrm{M}=1.69, \mathrm{SD}=0.54, \mathrm{~N}=75)$ and a mean workload level of 2.59 when performing the Baseline procedures $(\mathrm{M}=2.59, \mathrm{SD}=1.37, \mathrm{~N}=$ 75) as shown in Fig. 7. A Wilcoxon Test (i.e., a nonparametric within-subject test appropriate for analyzing two related samples of ordinal data) [12] was performed on the mean workload ratings and revealed that EPs reported experiencing a lower level of workload when they performed the

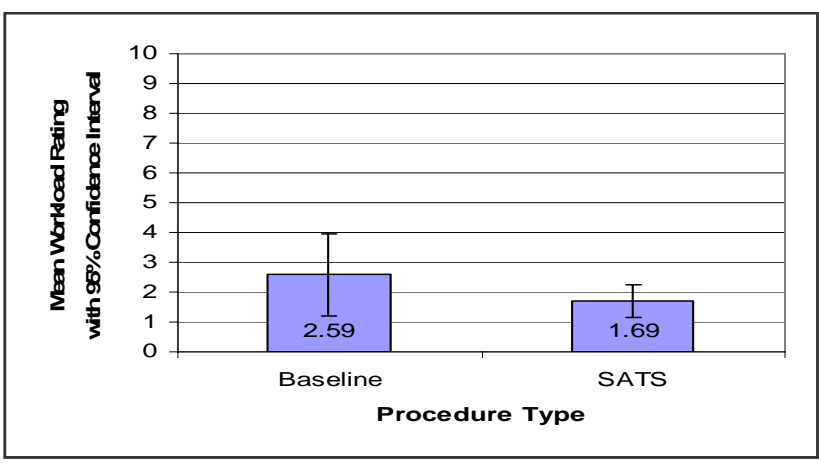

Figure 7. Mean Workload Rating SATS procedures than when they performed the Baseline procedures $(\mathrm{p}=0.05)$. This finding supports the hypothesis that pilots would not perceive an increase in workload when performing SATS procedures. 


\section{Subjective Assessments of Situation Awareness}

After each of the experiment's 10 test conditions, all EPs completed a SART questionnaire that included the three dimensions of demand, supply, and understanding as well as two independent dimensions of traffic awareness and navigation guidance. For the dimensions of demand, supply, and understanding, EPs used a scale ranging from 1 to 7 to report a score for each dimension. Global SART ratings were calculated by the formula: Situation Awareness $=$ Understanding - (Demand - Supply) and could range from 1 (representing a low level of SA) to 14 (representing a high level of SA). In the current study, calculated SART ratings ranged from 3 to 13 . For traffic awareness and navigation guidance awareness, scores ranging from 2 to 7 on a scale of 1 (low) to 7 (high) were collected from the EPs. As reported below, Wilcoxon Tests were used to analyze the SART, traffic awareness, and navigation guidance awareness scores.

A mean SART rating of 9.6 was calculated for the SATS procedures $(\mathrm{M}=9.6, \mathrm{SD}=1.97, \mathrm{~N}=75)$, and a mean SART rating of 8.05 was calculated for the Baseline procedures $(\mathrm{M}=8.05, \mathrm{SD}=2.68, \mathrm{~N}=75)$ as

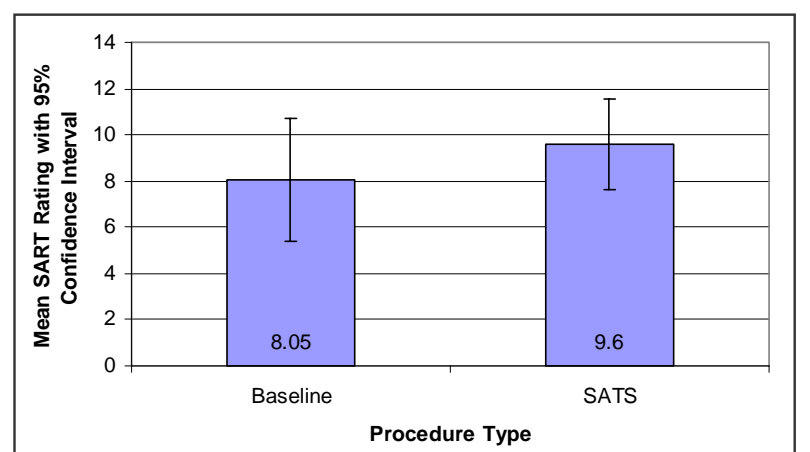

Figure 8. Mean SART Rating shown in Fig. 8. The results of a Wilcoxon Test indicated that, at a statistically significant level, the SART ratings associated with the performance of the SATS procedures were higher than those associated with the performance of the Baseline procedures $(\mathrm{p}=0.02)$.

\section{Subjective Assessment of Traffic Awareness}

With respect to traffic awareness, a mean rating of 6.55 was calculated for the SATS procedures $(\mathrm{M}=$ $6.55, \mathrm{SD}=0.72, \mathrm{~N}=75)$, and a mean rating of 5.59 was calculated for the Baseline procedures $(\mathrm{M}=5.59$, $\mathrm{SD}=1.43, \mathrm{~N}=75$ ) as shown in Fig. 9. A Wilcoxon Test revealed that a statistically significant difference was found to exist between the mean traffic awareness rating for the SATS procedures and the mean traffic awareness rating for the Baseline procedures $(p=0.0003)$ as shown in Fig. 9. This finding indicates that EPs reported experiencing higher levels of traffic awareness when they performed the SATS procedures than when they performed the Baseline procedures. Therefore, the

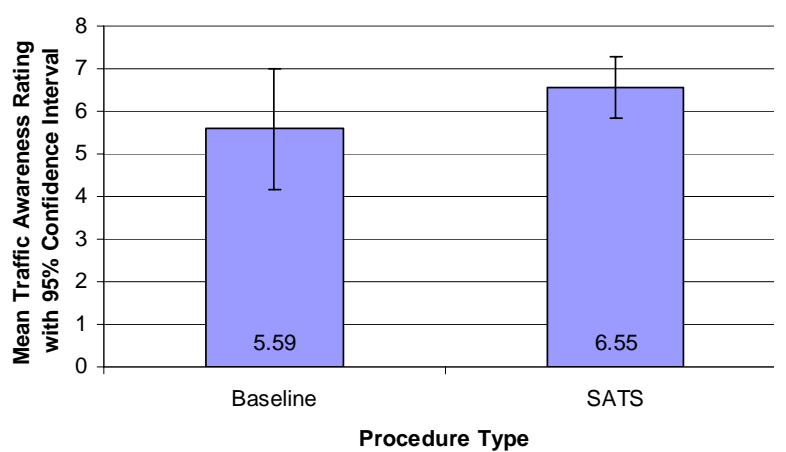

Figure 9. Mean Traffic Awareness Rating hypothesis that pilots would not experience a decrease in situation awareness when performing SATS procedures was supported.

\section{E. Subjective Assessment of Navigation Guidance Awareness}

With respect to navigation guidance SA, a mean rating of 6.45 was calculated for the SATS procedures $(\mathrm{M}=6.45, \mathrm{SD}=0.65, \mathrm{~N}=75)$, and a mean rating of 5.44 was calculated for the Baseline procedures $(\mathrm{M}=5.44, \mathrm{SD}=1.30, \mathrm{~N}=75)$ as shown in Fig. 10. A Wilcoxon Test revealed that a statistically significant difference was found to exist between the mean navigation guidance awareness rating for the SATS procedures and the mean navigation guidance awareness rating for the Baseline procedures $(p=0.001)$. This finding indicates that EPs reported higher levels of

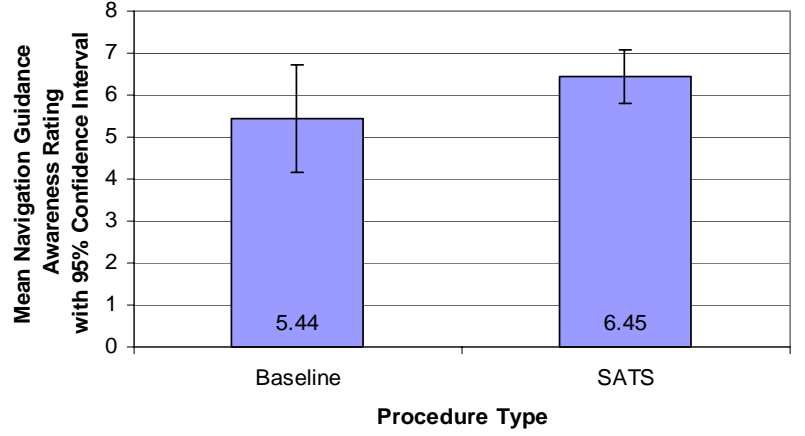

Figure 10. Mean Navigation Guidance Awareness 
navigation guidance awareness when they performed the SATS procedures than when they performed the Baseline procedures. Again, the hypothesis that pilots would not experience a decrease in situation awareness when performing SATS procedures was supported.

\section{F. Usability Questionnaires}

EPs were asked a number of questions regarding the SATS HVO procedures and the experimental interface designed for the simulation experiment. Table 1 shows the procedure based questions and the summary of the responses given by the EPs. Overall, all of the EPs found the procedures easy to follow and not more demanding then current procedures.

Table 1: Summary of Responses to Pilot Usability Questionnaires

\begin{tabular}{|l|l|l|}
\hline & Question & Summary of Responses \\
\hline 1 & $\begin{array}{l}\text { How easy was it to follow the SATS HVO flight } \\
\text { procedures? }\end{array}$ & $\begin{array}{l}100 \% \text { of EPs responded "Easy" to "Very Easy" } \\
\text { to follow. }\end{array}$ \\
\hline 2 & $\begin{array}{l}\text { If you found that the SATS HVO flight procedures were } \\
\text { difficult to follow, was the difficultly associated with the } \\
\text { procedures a result of: (turn into the form of a question) }\end{array}$ & $\begin{array}{l}100 \% \text { of EPs responded either no score or not } \\
\text { applicable. }\end{array}$ \\
\hline 3 & $\begin{array}{l}\text { How complicated were the SATS HVO flight procedures } \\
\text { compared to conventional flight procedures? }\end{array}$ & $\begin{array}{l}\text { All EPs rated the SATS HVO procedures as being } \\
\text { between "Equally Complicated to" and } \\
\text { "Significantly Less Complicated than" } \\
\text { conventional flight procedures. }\end{array}$ \\
\hline 4 & $\begin{array}{l}\text { Was the holding procedure required within the SCA more } \\
\text { difficult than the holding procedure required during } \\
\text { conventional IFR? }\end{array}$ & $\begin{array}{l}\text { 93\% of EPs responded "No" and 7\% responded } \\
\text { "Somewhat" }\end{array}$ \\
\hline 5 & $\begin{array}{l}\text { How straightforward was the sequencing information } \\
\text { provided during the SATS HVO scenarios? }\end{array}$ & $\begin{array}{l}73 \% \text { of EPs responded "Very Straight-Forward" } \\
\text { and 27\% responded "Straight-Forward" }\end{array}$ \\
\hline 6 & $\begin{array}{l}\text { During the SAT HVO approaches, how useful was the } \\
\text { "time to approach (TTA)" information? }\end{array}$ & $\begin{array}{l}53 \% \text { of EPs responded "Very Useful" and 47\% } \\
\text { responded "Useful" }\end{array}$ \\
\hline
\end{tabular}

\section{Summary}

This paper provides a description of a simulation experiment, that NASA Langley Research Center conducted as part of the SATS HVO concept validation process to determine pilot acceptability of normal HVO procedures. In addition, an in-depth description of the experiment's results is provided that includes flight Technical Error (FTE) and subjective pilot ratings of workload and situation awareness.

The comparative FTE analysis described includes lateral, vertical, and airspeed deviations from the Baseline and SATS HVO experimental flight procedures. The subjective pilot rating results are from MCH workload assessment ratings, SART ratings of situation awareness. Usability questionnaires were also given to pilots to assess their views on specific procedure issues.

Based on FTE analysis, all evaluation subjects, low-time instrument-rated pilots, flew the HVO procedures safely and proficiently and with no more workload in comparison to today's system (Baseline). Specifically, subject pilots maintained airspeed and lateral path more accurately when they performed the SATS scenarios than when they performed the Baseline scenarios. Subjects maintained altitude equally well in both SATS and Baseline scenarios.

Pilots assessed their workload to be less with SATS HVO than Baseline scenarios, and situation awareness, traffic awareness, and navigation guidance awareness was greater with SATS than Baseline scenarios.

The analysis of results presented in this paper indicate that for all the tested conditions, the answer to the questions "Can pilots safely and proficiently fly an airplane while performing SATS HVO procedures?" and "Do pilots perceive that workload, while performing HVO procedures, is no greater than flying in today's system?” is unequivocally positive. The same research objectives and similar results were obtained by a flight test conducted by NASA in the summer of 2004. Other research activities followed this experiment that included piloted studies of non-normal procedures, Monte Carlo experiments and a full-system architecture test that was successfully shown to the public at the June 2005 SATS Technical Demonstration in Danville, VA. 


\section{References}

${ }^{1}$ Conway, S. and Consiglio, M., "A Method of Separation Assurance for Instrument Flight Procedures at Non-Radar Airports”, AIAA Guidance, Navigation and Control Conference, August 2003, Monterrey California.

${ }^{2}$ Jones, K., Williams, D., Consiglio M., Adams C., and Abbott T., "IFR Operations at Non-Towered, Non-Radar Airports: Can we do better than one at a time?”, 14th AIAA Lighter-Than-Air Systems Convention \& Exhibition, Akron, Ohio, July 16 18, 2001.

${ }^{3}$ Abbott T., Jones K., Consiglio M., Williams D., and Adams C., "Small Aircraft Transportation System, Higher Volume Operations Concept: Normal Operations”, NASA/TM-2004-213022.

${ }^{4}$ Abbott T., Consiglio M., Baxley B., Williams D., and Conway S., "Small Aircraft Transportation System, Higher Volume Operations Concept: Off-Nominal Operations, NASA/TM- 2005 (To be assigned).

${ }^{5}$ Magyarits S., Racine, N., Hadley, J., "Air Traffic Control Feasibility Assessment of Small Aircraft Transportation System (SATS) Higher Volume Operations (HVO),” DOR/FAA/CT-05/26, May 2005.

${ }^{6}$ Williams D., Consiglio M., Murdoch J., and Adams C., "Preliminary validation of the Small Aircraft Transportation Systems Higher Volume Operations (SATS HVO) Concept”, Proceedings of the 24th International Congress of the Aeronautical Sciences”, August 2004, Yokohama, Japan.

${ }^{7}$ Williams, D., Consiglio, M., Murdoch, J., and Adams, C., "Flight Technical Error Analysis of the Small Aircraft Transportation System Higher Volume Operations (SATS HVO) Simulation and Flight Experiments”, Proceeding of the 24th Digital avionics Systems Conference, October 30th, November 3rd, 2005. Arlington VA.

${ }^{8}$ Consiglio, M., Conway, S., Adams, C., and Syed, H.,"SATS HVO Procedures for Priority Landings and Mixed VFR/IFR Operations at Non-Towered, Non-Radar Airports “, Proceeding of the 24th Digital avionics Systems Conference, October 30th, November 3rd, 2005. Arlington VA.

${ }^{9}$ Adams, C., Consiglio, M., Conway, S., and Syed, H., "The Pilot Advisor: Assessing the Need for a Procedural Advisory Tool”, Proceeding of the 24th Digital avionics Systems Conference, October 30th, November 3rd, 2005. Arlington VA.

${ }^{10}$ Williams, D., Murdoch, J., Adams C., Consiglio, M. Sturdy J. and Peplow K., "The Small Aircraft Transportation System Higher Volume Operations (SATS HVO) Flight Experiment using NASA's Cirrus SR22”. Aviation Technology Integration and Operations Conference, 26 - 28 Sep 2005, Hyatt Regency Crystal City, Arlington, Virginia.

${ }^{11}$ Endsley, M. R., Selcon, S. J., Hardiman, T. D., \& Croft, D. G. (1998). “A comparative analysis of SAGAT and SART for evaluations of situation awareness”. Proceedings of the 42nd Annual Meeting of the Human Factors \& Ergonomics Society, USA, 82-86.

${ }^{12}$ Norusis, M. J. (1993). SPSS ${ }^{\circledR}$ for Windows ${ }^{\mathrm{TM}}$ : Base system user’s guide, release 6.0. Chicago, IL: SPSS Inc.

${ }^{13}$ Wierwille, W. W., \& Casali, J. G. (1983). A valid rating scale for global mental workload measurement. Proceedings of the Human Factors Society 27th Annual Meeting (pp. 129-133).

${ }^{14}$ FAA-S-8081-4D, 2004, Instrument Rating Practical Test Standards for Airplane, Helicopter, Powered Lift.”

${ }^{15}$ Keppel, G., 1991, Design and Analysis: A Researcher’s Handbook. Third ed., Prentice-Hall, Inc. 